\title{
Outcome of tricuspid valve surgery in the presence of permanent pacemaker
}

\author{
Nishant Saran, MBBS, ${ }^{a}$ Sameh M. Said, MD,${ }^{\text {a }}$ Hartzell V. Schaff, MD, ${ }^{a}$ Simon Maltais, MD, ${ }^{\mathrm{a}}$

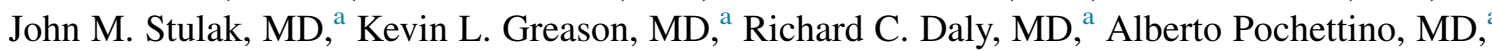 \\ Katherine S. King, MS, ${ }^{b}$ and Joseph A. Dearani, MD $^{\mathrm{a}}$
}

\section{ABSTRACT}

Objectives: Given the paucity of available literature, we sought to evaluate the mechanisms of tricuspid regurgitation and the outcomes of tricuspid valve surgery in the presence of permanent pacemakers.

\begin{abstract}
Methods: We retrospectively reviewed the records of 622 adult patients who underwent tricuspid valve surgery in the presence of permanent pacemakers between January 1993 and December 2013. Those with prosthetic tricuspid valve or tricuspid valve endocarditis and those undergoing concomitant heart transplant were excluded $(\mathrm{n}=23)$. Patients were divided into 2 etiologic groups: pacemaker-associated tricuspid regurgitation $(\mathrm{n}=349,58 \%)$ and pacemaker-induced tricuspid regurgitation $(n=249,42 \%)$. One patient was not categorized, because permanent pacemaker involvement was unknown.
\end{abstract}

Results: Mean age was $69.5 \pm 12.0$ years; 312 patients (52\%) were female. In pacemaker-associated tricuspid regurgitation, the most common cause was functional $(\mathrm{n}=304,87 \%)$. The most common mechanism leading to pacemaker-induced tricuspid regurgitation was restricted leaflet mobility $(\mathrm{n}=101,41 \%)$, followed by adherent leaflet to the leads $(\mathrm{n}=93,37 \%)$, leaflet perforation $(\mathrm{n}=30,12 \%)$, scarring of leaflets $(\mathrm{n}=19,8 \%)$, and chordal entrapment $(\mathrm{n}=18,7 \%)$. The most common leaflet involved was septal leaflet $(\mathrm{n}=182,73 \%)$. Tricuspid valve repair $(\mathrm{n}=215,62 \%)$ was higher in the pacemaker-associated tricuspid regurgitation group. In multivariable analysis, pacemaker-induced tricuspid regurgitation was found to be protective with improved survival (hazard ratio [HR], 0.79; 95\% confidence interval [CI], 0.68-0.98). Other independent risk factors of mortality included tricuspid valve replacement (HR, 1.50; 95\% CI, 1.20-1.87), nonelective surgery (HR, 1.66; 95\% CI, 1.33-2.08), diabetes (HR, 1.37; 95\% CI, 1.09-1.73), severe tricuspid regurgitation (HR, 1.42; 95\% CI, 1.04-1.95), and older age when there was a concomitant aortic valve surgery (HR, 1.44; 95\% CI, 1.15-1.79).

Conclusions: Several mechanisms lead to pacemaker-induced tricuspid regurgitation. Pacemaker-induced tricuspid regurgitation when compared with pacemaker-associated tricuspid regurgitation carries a better prognosis with improved survival. (J Thorac Cardiovasc Surg 2018;155:1498-508)

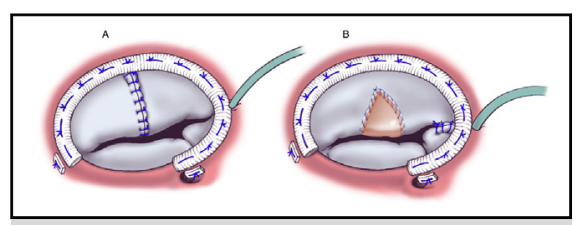

TV repair in patients with lead-induced TR.

\section{Central Message}

Several mechanisms lead to TR in patients with PPMs undergoing TV surgery. Patients with lead-induced TR have better survival.

\section{Perspective}

There are limited data about mechanisms of TR and outcomes of TV surgery in patients with PPMs. This study finds that patients with lead-induced TR have better survival. Alternatives to endocardial pacing lead systems, such as leadless/epicardial pacing systems, may be worth considering to avoid PPM lead-induced TR.

See Editorial Commentary page 1509.

See Editorial page 1496.
Tricuspid regurgitation (TR) is a common valvular lesion. It is functional in nature or due to intrinsic valvular pathology. Trauma to the tricuspid valve (TV) by transvenous leads of a permanent pacemaker (PPM) is a known iatrogenic cause

From the Departments of a Cardiovascular Surgery, and ${ }^{\mathrm{b}}$ Biomedical Statistics and Informatics, Mayo Clinic, Rochester, Minn.

Read at the 97th Annual Meeting of The American Association for Thoracic Surgery, Boston, Massachusetts, April 29-May 3, 2017.

Received for publication April 28, 2017; revisions received Oct 21, 2017; accepted for publication Nov 17, 2017; available ahead of print Feb 3, 2018. of significant TR. ${ }^{1}$ Paniagua and colleagues ${ }^{2}$ found the prevalence of moderate to severe TR in patients with PPM leads is approximately $25 \%$, more than twice the prevalence they found among patients without leads. Other

\footnotetext{
Address for reprints: Sameh M. Said, MD, Department of Cardiovascular Surgery, Mayo Clinic, 200 First St SW, Rochester, MN 55905 (E-mail: Said.sameh@ mayo.edu). $0022-5223 / \$ 36.00$

Copyright () 2017 by The American Association for Thoracic Surgery https://doi.org/10.1016/j.jtcvs.2017.11.093
} 


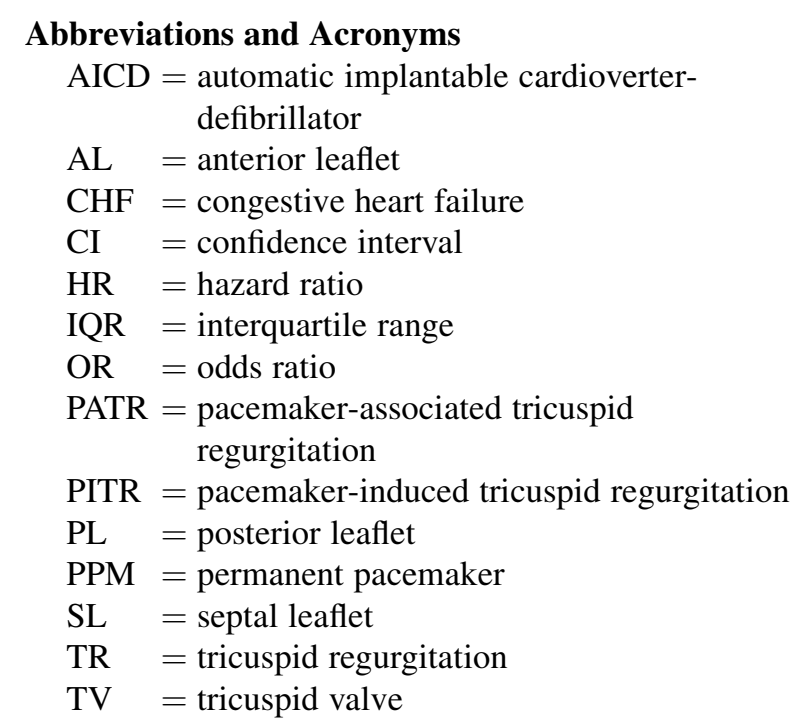

(4) Scanning this QR code will take you to the supplemental figures and table. To view the AATS Annual Meeting Webcast, see the URL next to the webcast thumbnail.

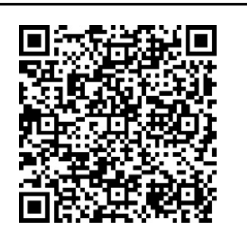

studies have found a higher prevalence of TR in patients with PPM. ${ }^{3,4}$ However, there are limited data available regarding the mechanisms leading to TR in patients with PPM and the outcome of TV surgery, either as repair or replacement, in such patients. Our objective in this study is to identify the various mechanisms leading to TR in patients with PPM and evaluate the outcome of TV surgery in these patients.

\section{PATIENTS AND METHODS}

We retrospectively reviewed the charts of 622 consecutive adult patients who underwent TV surgery in the presence of PPM with endocardial leads between January 1993 and December 2013 at our institution. The study was approved by the institutional review board at Mayo Clinic, Rochester. Patients with TV endocarditis $(n=7)$, patients with prosthetic TV $(\mathrm{n}=12)$, and patients undergoing concomitant heart transplantation $(n=4)$ were excluded. The remaining patients $(n=599)$ who underwent TV surgery in the presence of PPM had moderate/severe TR identified on preoperative transthoracic or intraoperative transesophageal echocardiography and were graded per the American Society of Echocardiography Guidelines..$^{5}$ Baseline patient characteristics were obtained from review of the medical record and reported on the basis of definitions set forth by the Society of Thoracic Surgeons Adult Cardiac Database. ${ }^{6}$

The role of PPM leads in contributing to TR was based on operative findings. Patients were divided into 2 groups: (1) pacemaker-associated
TR (PATR) $(\mathrm{n}=349,58 \%)$, in which patients had significant TR not found to be caused by PPM leads; and (2) pacemaker-induced TR (PITR) ( $\mathrm{n}=249,42 \%$ ), in which PPM leads directly contributed to the development of significant TR. In 1 patient it could not be ascertained if PPM contributed to the development of TR, and was thus excluded from the study $(\mathrm{n}=598)$. PITR was defined when PPM leads contributed to TR through the following mechanisms as noted in the operative findings (1) restriction of the mobility of otherwise normal-appearing leaflets or subvalvular apparatus; (2) scarring or fusion of leaflets to each other; (3) leaflet adherence to the leads; (4) leaflet perforation; or (5) chordal entrapment (Figure 1).

\section{Operative Approach}

Patients underwent TV repair $(n=318)$ or TV replacement $(n=280)$. The approach to TV surgery varied with individual surgeon preferences. Repair was attempted when the valve was not extensively damaged. Repair techniques included (1) detaching and moving away adherent leads; (2) suture repair of leaflets; (3) pericardial patch repair of perforated leaflets; and (4) translocating leads to the recess at posteroseptal or anteroposterior commissures with suture fixation. ${ }^{7}$ Mostly autologous pericardial patches were used for leaflet repair, although the CorMatrix (CorMatrix Cardiovascular, Inc, Atlanta, Ga) membrane was used occasionally. Suture annuloplasty was performed in 145 subjects $(46 \%)$, ring annuloplasty was performed in 171 subjects $(54 \%)$, and 2 subjects did not require annuloplasty (Figure 2). Post-TV repair less than moderate TR was accepted as a successful result, with all patients exiting the operating room with less than moderate TR. Patients receiving TV repair were discharged only if they had less than moderate TR on predismissal echocardiogram. When replacement was done, valve prostheses (mechanical, $\mathrm{n}=48,21 \%$; and bioprosthesis, $\mathrm{n}=232,79 \%$ ) were implanted with an attempt to avoid excising the leaflets and to preserve the entire valvular apparatus. In some mechanical valve implantations, the anterior leaflet (AL) was excised but the remaining valvular apparatus was preserved. In these cases, the lead was placed external to the sewing ring of the prosthesis. ${ }^{7,8}$

\section{Statistical Analysis}

Descriptive statistics for continuous variables are presented as mean \pm standard deviation or median (interquartile range [IQR]) where appropriate and categoric variables as $(\mathrm{n}=$ count, percentage). Analyses were performed in SAS v 9.4 (SAS Institute Inc, Cary, NC) with an alpha level of .05.

The reverse Kaplan-Meier estimate, where death is considered the censoring event, was used to estimate median (IQR) follow-up time. Survival and incidence of reoperation were estimated with the Kaplan-Meier product-limit method with $95 \%$ confidence intervals (CIs) on all figures. Proportional hazard models were used to measure the effect of baseline characteristics on survival. A multivariable model was developed using purposeful selection to assess the effect of TV surgery and PITR on survival while controlling for confounding factors. ${ }^{9}$ Variables were included in the initial model if the univariate significance was less than .20 , and they remained in the model at this alpha level. All pairwise interactions were considered, and the linearity of continuous variables was assessed with splines.

Logistic regression was used to assess the relationship of baseline characteristics with etiology (Table 1). The pacemaker etiology cohorts demonstrated differences on several important preoperative characteristics, suggesting possible inherent differences in the cohorts. In a sensitivity analysis, we balanced the covariates between the etiology groups and then evaluated the etiology effect in the equalized cohorts for comparison with the multivariable model. A propensity score predicting pacemaker involvement was computed from a multivariable logistic model developed through purposeful selection using the characteristics in the Table 2. PITR subjects were then matched to PATR subjects on the logit scale of the propensity 

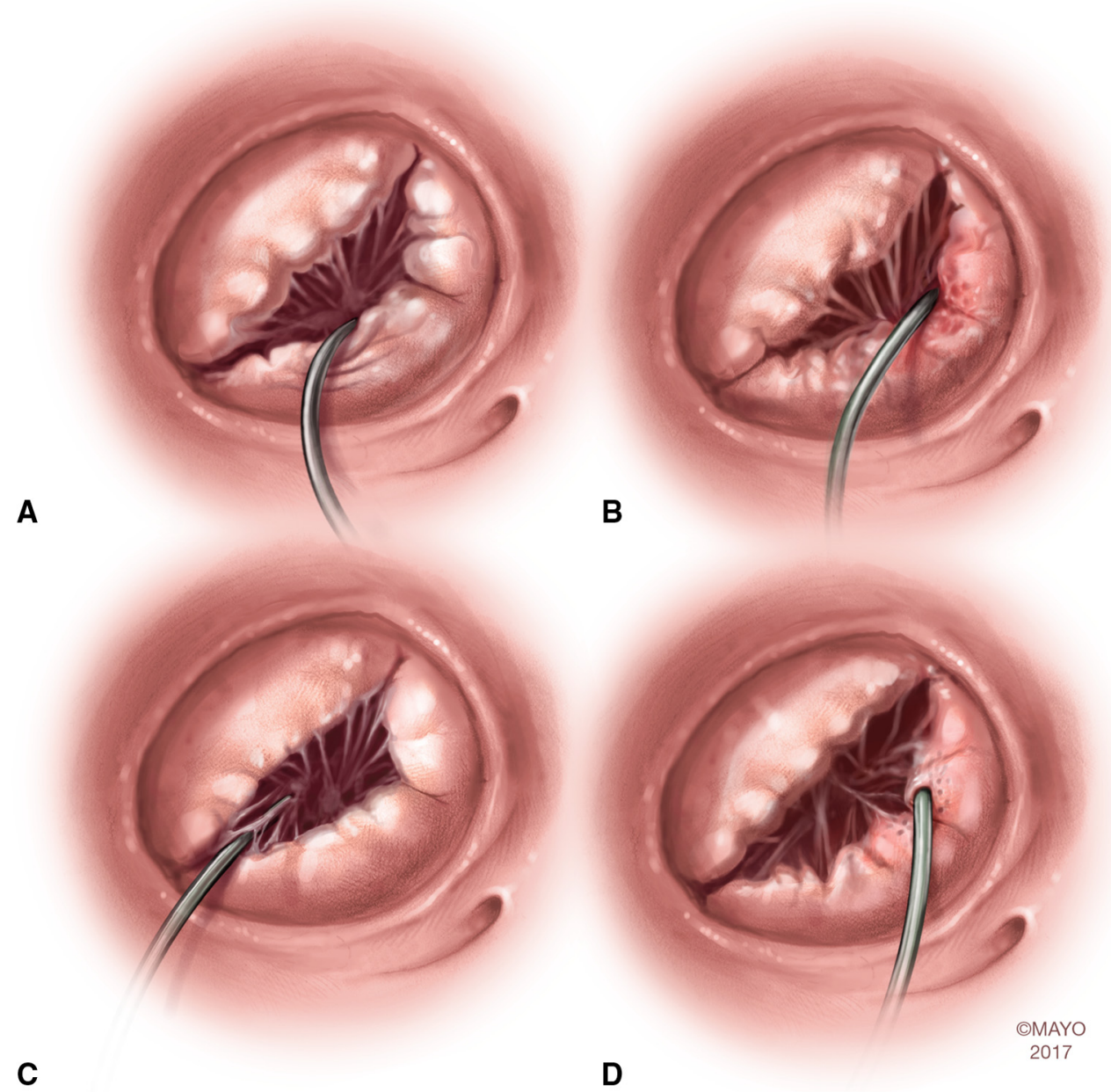

FIGURE 1. A, Restricted mobility of SL. B, Scarring and fusion of PLs and SLs. C, Chordal entrapment. D, PL perforation.

scores using a caliber of 0.2 of the standard deviation of the logit. ${ }^{10}$ This resulted in 203 matched pairs of PITR and PATR subjects well balanced on most covariates (Figure E1). The proportional hazard model was used to estimate the effect of pacemaker etiology in the matched cohort.

Because TV replacement was found to be statistically associated with mortality, multivariable logistic regression was used to identify independent risk factors for TV replacement in a supplemental analysis. Mechanisms of TR and leaflet involvement in the PITR cohort were additionally analyzed.

\section{RESULTS}

Baseline characteristics of the 2 groups, PATR and PITR, are shown in Table 1 . The 2 groups differed from each other with regard to congestive heart failure (CHF), which was more common in the PATR group (odds ratio [OR], 1.50; $95 \%$ CI, 1.07-2.12; $P=.020)$. PITR was more commonly found in the second half (2004-2013) of this 20-year study $(P=.032)$.

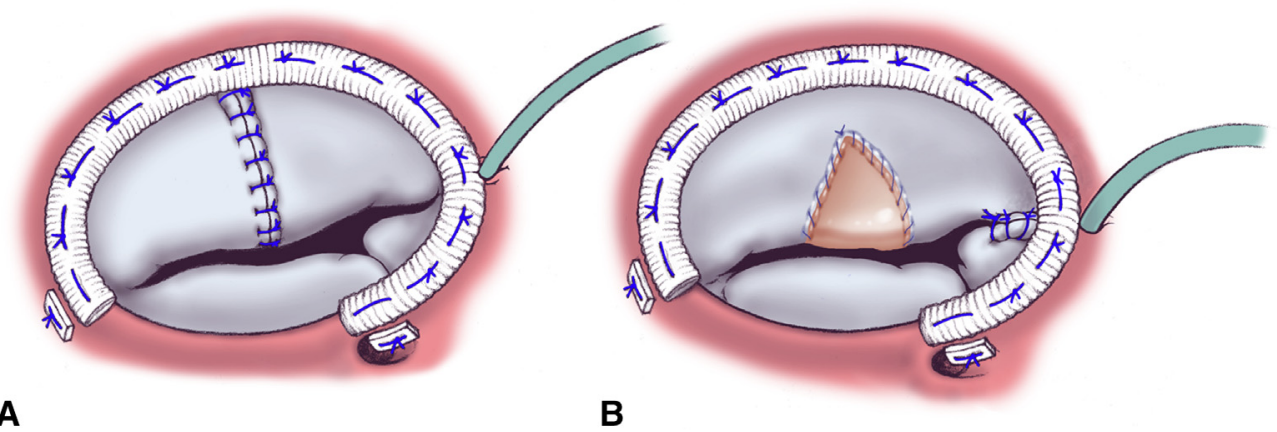

FIGURE 2. A, Detaching lead with suture repair. B, Pericardial patch repair with lead translocation. 
TABLE 1. Characteristics of the study cohort

\begin{tabular}{|c|c|c|c|}
\hline Preoperative & $\operatorname{PATR}(\mathbf{N}=349)$ & $\operatorname{PITR}(N=249)$ & $P$ value \\
\hline Age at surgery & $69.5(12.0)$ & $69.6(12.0)$ & .922 \\
\hline Pulmonary artery systolic pressure & $50.4(17.3)$ & $49.8(16.6)$ & .639 \\
\hline Decade (2004-2013) & $271(77.7 \%)$ & $211(84.7 \%)$ & .032 \\
\hline Female & $176(50.4 \%)$ & $136(54.6 \%)$ & .312 \\
\hline Nonelective surgery & $114(32.7 \%)$ & $81(32.5 \%)$ & .972 \\
\hline Ejection fraction $<40 \%$ & $84(24.1 \%)$ & $49(19.8 \%)$ & .206 \\
\hline NYHA Class III/IV & $300(87.0 \%)$ & $218(87.9 \%)$ & .732 \\
\hline Cardiogenic shock & $5(1.4 \%)$ & $2(0.8 \%)$ & .487 \\
\hline Hypertension & $216(61.9 \%)$ & $160(64.3 \%)$ & .555 \\
\hline Diabetes & $81(23.2 \%)$ & $55(22.1 \%)$ & .748 \\
\hline Cerebrovascular accident & $38(10.9 \%)$ & $21(8.4 \%)$ & .322 \\
\hline Coronary artery disease & $158(45.7 \%)$ & $112(45.0 \%)$ & .869 \\
\hline Dialysis & $8(2.3 \%)$ & $4(1.6 \%)$ & .558 \\
\hline Atrial fibrillation/flutter/SVT & $199(57.0 \%)$ & $144(57.8 \%)$ & .843 \\
\hline CHF & $245(70.2 \%)$ & $152(61.0 \%)$ & .020 \\
\hline Previous TV surgery (repair) & $17(4.9 \%)$ & $7(2.8 \%)$ & .212 \\
\hline Previous aortic valve surgery & $65(18.6 \%)$ & $34(13.7 \%)$ & .108 \\
\hline Previous mitral valve surgery & $100(28.7 \%)$ & $64(25.7 \%)$ & .425 \\
\hline Previous pulmonary valve surgery & $0(0.0 \%)$ & $1(0.4 \%)$ & .986 \\
\hline Previous CABG & $82(23.5 \%)$ & $61(24.5 \%)$ & .776 \\
\hline Previous valve or CABG & $187(53.6 \%)$ & $124(49.8 \%)$ & .362 \\
\hline \multicolumn{4}{|l|}{ Operative } \\
\hline Crossclamp time (min) & $61.0(39.0,93.0)$ & $46.5(27.0,82.0)$ & .020 \\
\hline Perfusion time (min) & $104.0(74.0,150.0)$ & $88.0(63.0,128.0)$ & $<.001$ \\
\hline TV replacement & $134(38.4 \%)$ & $146(58.6 \%)$ & $<.001$ \\
\hline Isolated TV surgery & $37(10.6 \%)$ & $55(22.1 \%)$ & $<.001$ \\
\hline Concomitant mitral valve surgery & $171(49.0 \%)$ & $71(28.5 \%)$ & $<.001$ \\
\hline Concomitant aortic valve surgery & $74(21.2 \%)$ & $43(17.3 \%)$ & .233 \\
\hline Concomitant pulmonary valve surgery & $3(0.9 \%)$ & $1(0.4 \%)$ & .509 \\
\hline Concomitant CABG & $54(15.5 \%)$ & $36(14.5 \%)$ & .734 \\
\hline Concomitant arrhythmia surgery & $31(8.9 \%)$ & $23(9.2 \%)$ & .881 \\
\hline \multicolumn{4}{|l|}{ Postoperative } \\
\hline ICU stay (h) & $73(42,199)$ & $66(26,120)$ & 620 \\
\hline Length of stay (d) & $10(7,19)$ & $9(6,14)$ & .202 \\
\hline Renal failure & $74(21.2 \%)$ & $34(13.7 \%)$ & .019 \\
\hline Low output cardiac syndrome & $25(7.2 \%)$ & $12(4.8 \%)$ & .244 \\
\hline ECMO support & $3(0.9 \%)$ & $2(0.8 \%)$ & .922 \\
\hline 30-d mortality & $33(9.5 \%)$ & $11(4.4 \%)$ & .023 \\
\hline Readmission with $30 \mathrm{~d}$ of surgery & $39(12.2 \%)$ & $32(13.7 \%)$ & .615 \\
\hline
\end{tabular}


TABLE 2. Baseline characteristics in the propensity-matched cohort

\begin{tabular}{|c|c|c|c|}
\hline Variables & $\operatorname{PATR}(N=203)$ & $\operatorname{PITR}(\mathbf{N}=203)$ & $P$ value \\
\hline Age at surgery, y & $69.0(12.2)$ & $69.4(12.0)$ & .853 \\
\hline Pulmonary artery systolic pressure & $49.1(16.5)$ & $50.3(17.1)$ & .537 \\
\hline TV replacement & $110(54.2 \%)$ & $107(52.7 \%)$ & .765 \\
\hline Surgery year (2004-2013) & $170(83.7 \%)$ & $171(84.2 \%)$ & .892 \\
\hline Female & $95(46.8 \%)$ & $118(58.1 \%)$ & .022 \\
\hline Nonelective surgery & $65(32.0 \%)$ & $72(35.5 \%)$ & .463 \\
\hline Ejection fraction $<40 \%$ & $52(25.6 \%)$ & $40(19.8 \%)$ & .163 \\
\hline NYHA Class III/IV & $179(88.2 \%)$ & $176(86.7 \%)$ & .653 \\
\hline Cardiogenic shock & $4(2.0 \%)$ & $2(1.0 \%)$ & .411 \\
\hline Hypertension & $124(61.1 \%)$ & $129(63.5 \%)$ & .609 \\
\hline Diabetes & $49(24.1 \%)$ & $46(22.7 \%)$ & .725 \\
\hline Cerebrovascular accident & $19(9.4 \%)$ & $18(8.9 \%)$ & .863 \\
\hline Coronary artery disease & $88(43.8 \%)$ & $90(44.3 \%)$ & .911 \\
\hline Dialysis & $4(2.0 \%)$ & $4(2.0 \%)$ & 1.000 \\
\hline Atrial fibrillation/flutter/SVT & $110(54.2 \%)$ & $117(57.6 \%)$ & .484 \\
\hline Congestive heart failure & $141(69.5 \%)$ & $132(65.0 \%)$ & .341 \\
\hline Previous aortic valve surgery & $27(13.3 \%)$ & $31(15.3 \%)$ & .571 \\
\hline Previous mitral valve surgery & $52(25.6 \%)$ & $51(25.1 \%)$ & .909 \\
\hline Previous TV surgery (repair) & $8(3.9 \%)$ & $7(3.4 \%)$ & .793 \\
\hline Previous pulmonary valve surgery & $0(0.0 \%)$ & $1(0.5 \%)$ & .317 \\
\hline Previous CABG & $50(24.6 \%)$ & $45(22.2 \%)$ & .558 \\
\hline Any previous valve surgery or CABG & $99(48.8 \%)$ & $96(47.3 \%)$ & .766 \\
\hline Severe TR & $176(86.7 \%)$ & $182(89.7 \%)$ & .356 \\
\hline Isolated TV procedure & $35(17.2 \%)$ & $39(19.2 \%)$ & 607 \\
\hline Concomitant mitral valve surgery & $65(32.0 \%)$ & $69(34.0 \%)$ & .673 \\
\hline Concomitant aortic valve surgery & $40(19.7 \%)$ & $36(17.7 \%)$ & .611 \\
\hline Concomitant pulmonary valve surgery & $2(1.0 \%)$ & $1(0.5 \%)$ & .562 \\
\hline Concomitant $\mathrm{CABG}$ performed & $27(13.3 \%)$ & $30(14.8 \%)$ & .668 \\
\hline Concomitant arrhythmia surgery & $20(9.9 \%)$ & $16(7.9 \%)$ & .485 \\
\hline
\end{tabular}

PATR, Pacemaker-associated tricuspid regurgitation; PITR, pacemaker-induced tricuspid regurgitation; TV, tricuspid valve; NYHA, New York Heart Association; $S V T$, supraventricular tachycardia; $C A B G$, coronary artery bypass grafting; $T R$, tricuspid regurgitation.

\section{Operative Findings}

The most common etiology in the PATR group was functional TR $(n=304,87 \%)$. In the PITR group, the most common mechanism leading to TR was restricted mobility of leaflets, followed by adherent leaflet to the leads, leaflet perforation, scarring of leaflets, and chordal entrapment. The most common leaflet involved was the septal leaflet (SL) $(\mathrm{n}=182,73 \%)$, followed by the posterior leaflet $(\mathrm{PL})(\mathrm{n}=129,52 \%)$ and $\mathrm{AL}(\mathrm{n}=103,41 \%)$. All 3 leaflets were involved in 84 patients (34\%) (Table 3 ).

There were 15 patients in whom the endocardial pacemaker leads were excised and substituted with epicardial leads. There were 144 patients $(24.1 \%)$ with an automatic implantable cardioverter-defibrillator (AICD) lead in addition to PPM. There was no significant difference between patients with and without AICD leads in terms of increased PITR, as $41.4 \%$ of subjects without AICD leads had PITR and $42.36 \%$ of subjects with AICD leads had PITR $(P=.84)$.

The majority of the patients had concomitant cardiac surgery performed besides TV surgery $(n=506,85 \%)$, with concomitant mitral valve surgery being the most common $(n=242,41 \%)$. The PATR group had a higher rate of concomitant mitral valve surgery, whereas isolated TV surgery was more likely in the PITR group. The PATR group had longer bypass $(P<.001)$ and crossclamp $(P=.02)$ times. With regard to the type of surgery, TV replacement was more likely in the PITR group, with 146 $(59 \%)$ receiving replacements in the PITR group and 134 $(38 \%)$ receiving replacements in the PATR group (Table 1). 
TABLE 3. Pacemaker-induced tricuspid regurgitation: Mechanism and leaflet involved

\begin{tabular}{|c|c|c|c|}
\hline Mechanism & $\begin{array}{c}\text { Leaflet } \\
\text { involved }\end{array}$ & $\begin{array}{l}\text { Additional leaflet/ } \\
\text { chordae if involved }\end{array}$ & Count \\
\hline \multirow{6}{*}{$\begin{array}{l}\text { Restricted mobility } \\
\text { of leaflets } \\
\quad(\mathrm{n}=101,40.56 \%)\end{array}$} & All leaflets & & 78 \\
\hline & All leaflets & Chordal entrapment & 1 \\
\hline & Anterior & & 2 \\
\hline & Posterior & & 9 \\
\hline & Septal & & 10 \\
\hline & Septal & Chordal entrapment & 1 \\
\hline \multirow{4}{*}{$\begin{array}{l}\text { Adherent leaflet to lead } \\
\quad(\mathrm{n}=93,37.35 \%)\end{array}$} & Anterior & & 7 \\
\hline & Posterior & & 22 \\
\hline & Septal & & 54 \\
\hline & Septal & Chordal entrapment & 10 \\
\hline \multirow{3}{*}{$\begin{array}{l}\text { Leaflet perforation } \\
\qquad(\mathrm{n}=30,12.05 \%)\end{array}$} & Anterior & & 7 \\
\hline & Posterior & & 6 \\
\hline & Septal & & 17 \\
\hline \multirow{6}{*}{$\begin{array}{l}\text { Scarring of leaflets } \\
\quad(\mathrm{n}=19,7.63 \%)\end{array}$} & All leaflets & & 5 \\
\hline & Anterior & & 1 \\
\hline & Posterior & & 5 \\
\hline & Septal & & 5 \\
\hline & Anterior & Posterior & 2 \\
\hline & Septal & Posterior & 1 \\
\hline $\begin{array}{l}\text { Chordal entrapment } \\
\qquad(\mathrm{n}=6,2.41 \%)^{*}\end{array}$ & Chordae & & 6 \\
\hline
\end{tabular}

*Chordal entrapment alone was present in 6 subjects; however, there were 12 other subjects in whom chordal involvement was in conjunction with other mechanisms. The total subjects with chordal involvement were $\mathrm{n}=18,7.23 \%$.

\section{Early Outcomes}

Operative mortality occurred in 44 subjects $(7.3 \%)$, with mortality in the PATR group $(n=33,9.5 \%)$ being more than twice the mortality found in the PITR group $(\mathrm{n}=11$, $4.4 \%, P=.023$ ) (Table 1 ). There was 1 subject who required a TV replacement within 30 days of the original TV repair.

\section{Follow-up and Late Outcomes}

The median follow-up time was 7.92 (IQR, 4.0-13.1) years with maximum follow-up time of 21.6 years. There were a total of only 14 reoperations with the incidence of reoperation at 1 and 10 years being $1 \%$ and $5 \%$, respectively (Kaplan-Meier incidence curve shown in Figure E2), whereas reoperation rates for repair patients were $0 \%$ and $5 \%$ at 1 and 10 years, respectively. Long-term mortality was univariately associated with age at surgery (hazard ratio [HR], 1.01, 95\% CI, 1.00-1.02;
$P=.006), \quad \mathrm{CHF}$ (HR, 1.41, 95\% CI, 1.13-1.75; $P=.002)$, coronary artery disease (HR, $1.40,95 \% \mathrm{CI}$, 1.14-1.71; $P=.001$ ), dialysis (HR, 2.07, 95\% CI, $1.07-4.02 ; P=.028$ ), diabetes (HR, $1.47,95 \% \mathrm{CI}$, $1.17-1.85 ; P<.001$ ), and nonelective surgery (HR, 1.48 , 95\% CI, 1.20-1.83; $P<.001)$ in patients with PPM undergoing TV surgery. Patients with TV replacement had worse survival compared with TV repair for the whole study group (HR, 1.31, 95\% CI, 1.07-1.60; $P=.008)$ and within the PITR and PATR cohorts. Overall, the PITR group seemed to have better survival compared with the PATR group (HR, 0.85, 95\% CI, 0.70-1.05; $P=.125$ ) (Figure 3). There was no interaction detected between the 2 etiology groups and the type of TV surgery performed with regard to survival $(P=.626)$. In addition, the mechanisms leading to TR and involvement of any specific leaflets/chordae were not associated with survival in the PITR group.

In multivariable analysis, it was found that PITR was still protective with an HR of 0.79 (95\% CI, 0.34-0.98). Other independent risk factors of mortality included TV replacement, nonelective surgery, diabetes, severe TR, and older age when there was a concomitant aortic surgery (Table 4). In the propensity-matched cohort of 203 pairs of PITR and PATR subjects (Table 2), PITR was still protective (HR, 0.78, 95\% CI, 0.61-1.00; $P=.052$ ) but not significant, although the HR was similar in magnitude to the multivariable adjusted HR.

\section{Choice of Surgery: Replacement Versus Repair}

The univariate logistic regression including all the preoperative and operative characteristics of the entire study group is shown in the Forest plot in Figure E3.

In the whole study group, multivariate analysis (Table E1) found PITR (OR, 2.14, 95\% CI, 1.44-3.18; $P<.001)$, severe TR (OR, 5.39, 95\% CI, 2.48-11.69; $P<.001$ ), CHF (OR, 1.90, 95\% CI, 1.21-2.98; $P=.005$ ), previous TV repair (OR, 13.16, 95\% CI, 3.19-54.24; $P<.001$ ), and age (OR for 10 years $1.19,95 \% \mathrm{CI}$, $1.00-1.40 ; P=.047$ ) to have higher odds of receiving TV replacement, whereas ejection fraction less than $40 \%$, concomitant mitral valve surgery, aortic valve surgery, coronary artery bypass grafting, and arrhythmia surgery were found to have greater odds of receiving TV repair.

In patients receiving PITR, univariate logistic regression found any SL involvement (OR, 4.07, 95\% CI, 2.07-7.94; $P<.001)$ or SL when involved alone (OR, $2.48,95 \% \mathrm{CI}$, 1.45-4.24; $P<.001)$ had better odds of successful repair. When the SL was not involved $(\mathrm{n}=67)$, only $13(19 \%)$ of those subjects received repair. Those with any chordal entrapment (OR, 3.82, 95\% CI, 1.08-13.54; $P=.038$ ) and PL involvement, if involved alone (OR, 3.61, 95\% CI, 1.59-8.16; $P=.002$ ), had higher odds of TV replacement. 

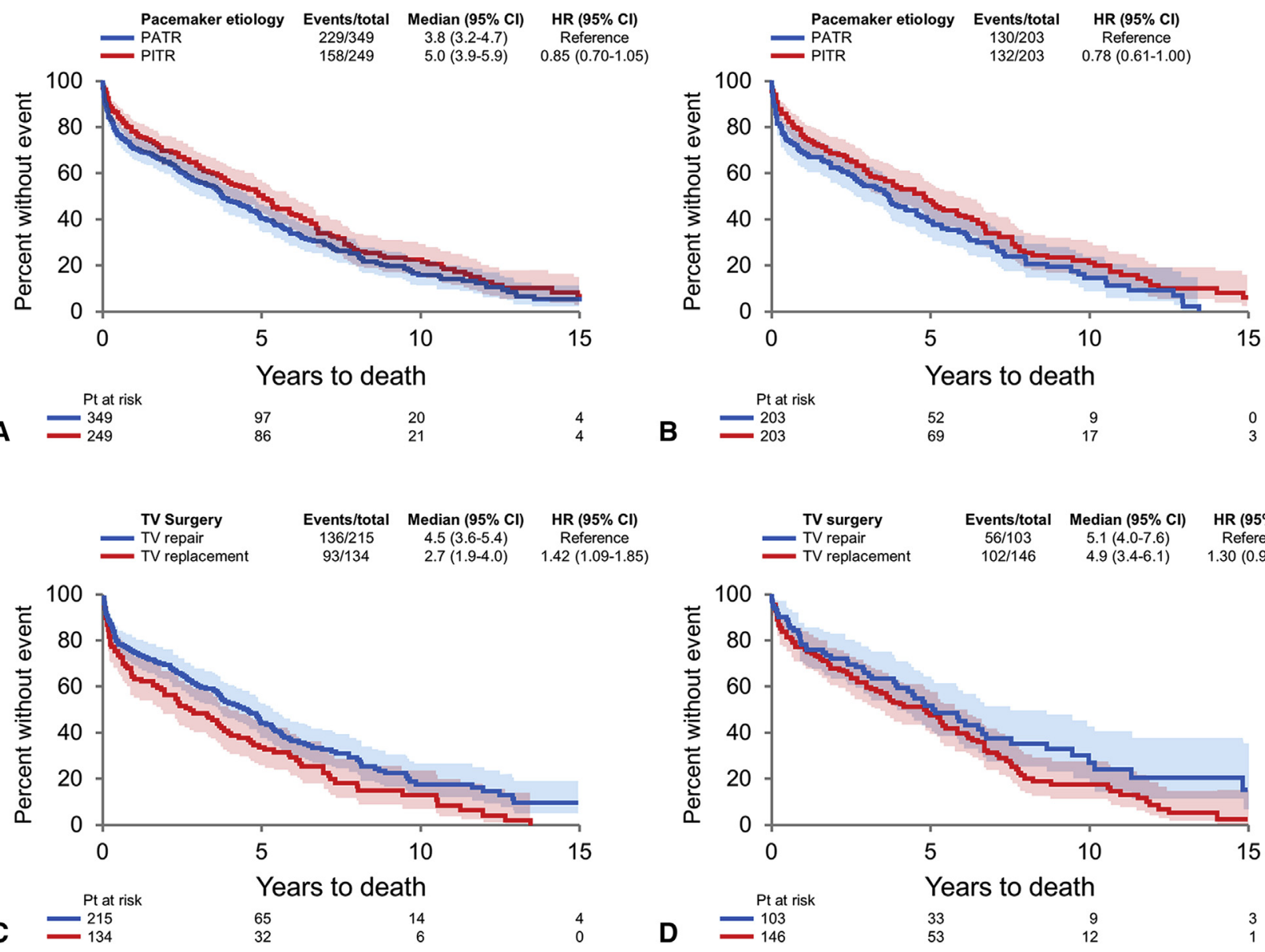

FIGURE 3. Kaplan-Meier survival curves for PITR versus PATR in the entire cohort (A), PITR versus PATR in the matched cohort (B), TV replacement versus repair in PATR (C), and TV replacement versus repair in PITR (D). CI, Confidence interval; HR, hazard ratio; PATR, pacemaker-associated tricuspid regurgitation; PITR, pacemaker-induced tricuspid regurgitation; $T V$, tricuspid valve.

\section{DISCUSSION}

In the present study, we reviewed our experience with TV surgery in the presence of PPM. We aimed to identify the mechanisms contributing to TR in patients with PPM and to evaluate the outcomes of TV surgery in this group. Of the 598 patients in the study, $249(42 \%)$ had PITR. Functional TR was the most common cause in the PATR group. In the PITR group, the most common mechanism involved was restricted mobility of leaflets and the most common leaflet involved was SL. PATR, TV replacement, severe TR, nonelective surgery, diabetes, and older age when associated with aortic valve replacement were found to be independent risk factors for mortality. The PITR cohort appeared to have better overall survival in comparison with the PATR group. PITR, severe TR, CHF, previous TV repair, and older age were associated with higher odds of receiving TV replacement.

Several studies have found an increased prevalence of TR in patients with PPM. ${ }^{1-3,11}$ Studies have also found an increase in TR grade in patients after PPM insertion., ${ }^{4,12}$ Klutstein and colleagues ${ }^{12}$ found that TR was worse by at least 2 grades in $18 \%$ of the patients who received PPM, although none required any surgical intervention. Patients who were older and who had left ventricular dysfunction were more likely to develop worsening TR grades. ${ }^{12}$ In 2003, Lin and colleagues ${ }^{13}$ found PPM lead-related injury to the TV can result in clinically important severe TR and secondary right heart failure. The most common mechanisms were leaflet impingement (39\%) followed by leaflet adherence $(34 \%)$, leaflet perforation $(17 \%)$, and lead entanglement $(9 \%)$. This is similar to our findings, with restricted mobility being the most common $(41 \%)$. However, we found an additional mechanism wherein there was scarring and fusion of leaflets $(8 \%)$. This was mostly found in the SL and PL with the lead still freely mobile within the TV. It seems even though the lead was freely mobile, the repeated contact and resulting friction with the TV apparatus during each cardiac cycle led to scarring and fusion of leaflets, and ultimately the lead might get adherent to the leaflets and entangled in the subvalvular apparatus. Right ventricular dyssynchrony as a possible functional cause for TR in patients paced with right ventricular leads 
TABLE 4. Risk factors for mortality in patients with permanent pacemaker undergoing tricuspid valve surgery (multivariable analysis)

\begin{tabular}{|c|c|c|c|c|}
\hline Risk factor & Reference & HR & $95 \%$ HR confidence limits & $P$ value \\
\hline Male & Female & 1.090 & $0.884-1.345$ & .420 \\
\hline Coronary artery disease & No & 1.228 & $0.987-1.529$ & .065 \\
\hline PITR & PATR & 0.791 & $0.637-0.982$ & .033 \\
\hline TV replacement & TV repair & 1.499 & $1.202-1.871$ & $<.001$ \\
\hline Nonelective surgery & Elective surgery & 1.662 & $1.328-2.081$ & $<.001$ \\
\hline Diabetes & No & 1.372 & $1.086-1.732$ & .008 \\
\hline Dialysis & No & 1.932 & $0.981-3.803$ & .057 \\
\hline Severe TR & Moderate & 1.423 & $1.036-1.953$ & .029 \\
\hline Age at surgery with concomitant aortic valve surgery & $10 \mathrm{y}$ & 1.436 & $1.154-1.787$ & Interaction: .023 \\
\hline Age at surgery without concomitant aortic valve surgery & $10 \mathrm{y}$ & 1.094 & $0.987-1.212$ & \\
\hline
\end{tabular}

HR, Hazard ratio; PITR, pacemaker-induced tricuspid regurgitation; PATR, pacemaker-associated tricuspid regurgitation; TV, tricuspid valve; TR, tricuspid regurgitation.

has been postulated. This abnormal sequence of pacing beginning in the apex may lead to delayed activation of the papillary muscle. ${ }^{2}$ We also found that PITR frequency was higher in the second decade of experience $(32.8 \%$ vs $43.8 \%$ ), which could be reflective of the more liberal use of PPM in the later decade.

In the present study, PITR was found to be an independent predictor for TV replacement. This is reflective of a difficult subset of patients to achieve a successful repair given the trauma and damage suffered by the TV due to endocardial leads. However, within the PITR group, of the 182 patients who had SL involvement, $90(49 \%)$ underwent successful repair $(P<.001)$, whereas when SL was not involved, only $19 \%$ had a successful repair. It is also interesting to note that when SL alone $(\mathrm{n}=86)$ was involved, it was still predictive of successful TV repair $(P<.001)$. The most common mechanism when the SL alone was involved was adherent leaflet to the lead $(\mathrm{n}=54,63 \%)$. It is important to note that neither restricted mobility nor adherent leaflet to the lead as mechanisms leading to TR was predictive of successful TV repair on their own. Nevertheless, it appears that if SL anatomy is well preserved, a successful TV repair is feasible. The preservation of subvalvular apparatus, it seems, is a prerequisite for a successful repair given that those with any chordal involvement had higher odds of getting TV replacement $(P=.038)$. Patients with PL involvement alone had a higher likelihood of getting the valve replaced $(P=.020)$. This is contrary to our expectations, because during TV repair we often sacrifice the PL and achieve bicuspidization to improve TV competency. It may be possible that a deformed PL, especially with scarring and fusion of adjacent leaflets or increased entanglement of the lead with the chords in this position, presents a situation that makes repair less feasible. Uehara and colleagues ${ }^{14}$ published a series of 6 patients wherein 1 patient having PL perforation was successfully repaired by incising the PL toward the annulus and then transferring and reaffixing the PPM lead to the annulus, followed by suture repair of the PL and De Vega's annuloplasty.

With several studies finding an increased prevalence of $\mathrm{TR}^{1-3,11}$ in patients with PPM with endocardial leads, alternative solutions such as epicardial/leadless pacemaker systems may offer some advantage by avoiding any damage to the TV. In our current practice, if endocardial leads appear to have excellent thresholds, we make an effort to preserve them or we excise them and place an epicardial system at the end. If the leads are to be preserved, we carefully detach them from the leaflet and reposition them against the TV annulus in the anteroposterior/posteroseptal commissure with a loose commissural stitch to prevent the leads from wandering into the TV orifice and interfering with leaflet function. A ring is applied at the completion of procedure. The lead is usually maintained outside the ring, but should be movable so that future removal by laser extraction, if needed, is not compromised. Likewise, if TV is being replaced, the lead is placed external to the prosthetic valve ensuring that it lies sufficiently mobile alongside the prosthesis. ${ }^{7}$ This is important because it has been shown that traumatic TR is not uncommon after percutaneous lead removals. ${ }^{15-18}$ Whenever possible, we avoid placing leads across bioprosthetic TVs. If a lead is placed inside the ring, it is ensured that it does not interfere with leaflet function.

There are limited data available on operative mortality and survival of those with PPMs with TR undergoing TV surgery. This lack of data is of particular concern especially when it has been shown that percutaneous lead removal for significant TR leads to infrequent improvement in TR grade. ${ }^{19}$ Lin and colleagues ${ }^{13}$ found an operative mortality of $2.4 \%$ for patients with lead-induced TR. In our study, 30-day mortality was higher at $4.4 \%$ in the PITR group and $9.5 \%$ in the PATR group. PATR had a higher proportion of patients with CHF, increased crossclamp and bypass times, and concomitant mitral valve surgery. This may be reflective of the more complicated nature of surgery in this group of patients and, 
thus, a higher early mortality. In the long term, PITR compared with PATR was found to be protective with improved survival (HR, 0.79; $P=.03$ ) (Table 3). The HR was similar in magnitude (HR, 0.78; $P=.05$ ) in the propensity-matched cohort, although we did see a difference in statistical significance. This could be due to subsetting in the propensity analysis, leading to a reduction in statistical power, therefore possibly resulting in a $P$ value greater than .05. The increased survival in the PITR cohort is plausible because pacemaker-induced TR is an iatrogenic cause restricted to TV that, when fixed, ensures the pathology stands corrected. In subjects with TR subsequent to left heart/inherent TV pathology, the disease process itself may be progressive enough to preclude better chances of survival. Severe TR, nonelective surgery, and older age when associated with aortic valve replacement were identified as other independent risk factors for mortality. This could be reflective of an advanced disease process leading to higher chances of mortality. Diabetes as an added comorbidity could also contribute to the worse survival in this group of patients.

Several studies have found TV repair to be associated with lower operative mortality and better survival compared with TV replacement, ${ }^{20-23}$ although others have found no significant difference. ${ }^{24}$ In our study, TV replacement was found to be an independent risk factor for mortality. Severe TR, age, CHF, and previous TV repair were identified as independent predictors of TV replacement, which is perhaps indicative of a more advanced disease process in patients who received TV replacement, possibly contributing to higher mortality. Because patients receiving PITR and patients undergoing TV repair have better survival, it may also be suggested that patients with chordal and PL involvement, which are predictive of TV replacement in PITR cases, if offered a repair, may gain a survival benefit.

\section{Study Limitations}

This study is a retrospective analysis from a single institute about patients with PPMs undergoing TV surgery. This precludes us from commenting about the incidence of the problem. We have included patients with functional TR, ischemic heart disease, and rheumatic heart disease. These may present as confounding factors contributing to the severity of TR, although we did not find any significant difference in pulmonary artery pressures between PATR and PITR. There is also a likely chance of right ventricle dyssynchrony leading to TR in patients who are paced through RV leads at apex, which we have not been able to exclude. The study cohorts are also heterogeneous, with the PATR group having a higher incidence of CHF and left heart interventions. However, we have tried to use appropriate statistical methods (multivariable and propensity-matched analysis) to best even out the differences. A multivariable analysis of the whole study group with regard to choice of surgery (repair/replacement) was done while controlling for possible confounders; however, our attempts at getting the same within the subsets of PITR and PATR were not feasible because of inadequate numbers within each subset cohort. Our data are limited with regard to long-term follow-up of TV repair, the failure of which may also affect survival. For a surrogate, we looked into the overall reoperation rates; however, low event count precluded any meaningful statistical exercise with regard to reoperation analysis. Last but not least, mechanisms causing TR in the current study were identified on the basis of intraoperative findings as described by the operating surgeon, and thus are subjective in nature, possibly contributing to a surgeon bias in deciding the approach toward the type of TV surgery.

\section{CONCLUSIONS}

Several mechanisms can lead to TR necessitating TV surgery in patients with PPM. Patients with TR due to lead injury from pacemakers tend to have better survival compared with those patients in whom TR is due to intrinsic valve/left heart pathology. Alternatives to endocardial pacing lead systems, such as leadless/epicardial pacing systems, may be worth considering to avoid PPM lead-induced TR.

\section{Webcast}

You can watch a Webcast of this AATS meeting presentation by going to: https://aats.blob.core.windows.net/media/17AM/ 2017-05-02/BallroomABC/05-02-17_BallroomABC_1635_ Saran.mp4.

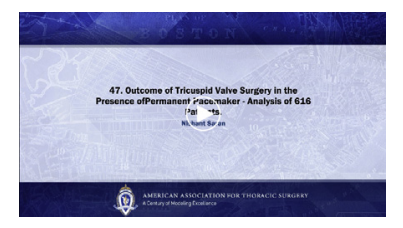

\section{Conflict of Interest Statement}

Authors have nothing to disclose with regard to commercial support.

\footnotetext{
References

1. Gibson TC, Davidson RC, DeSilvey DL. Presumptive tricuspid valve malfunction induced by a pacemaker lead: a case report and review of the literature. Pacing Clin Electrophysiol. 1980;3:88-95.

2. Paniagua D, Aldrich HR, Lieberman EH, Lamas GA, Agatston AS. Increased prevalence of significant tricuspid regurgitation in patients with transvenous pacemakers leads. Am J Cardiol. 1998;82:1130-9.

3. De Cock CC, Vinkers M, Van Campe LC, Verhorst PMJ, Viser CA. Long-term outcome of patients with multiple $(\geq 3)$ noninfected transvenous leads: a clinical and echocardiographic study. Pacing Clin Electrophysiol. 2000;23:423-6.

4. Kim JB, Spevack DM, Tunick PA, Bullinga JR, Kronzon I, Chinitz LA, et al. The effect of transvenous pacemaker and implantable cardioverter defibrillator lead placement on tricuspid valve function: an observational study. J Am Soc Echocardiogr. 2008;21:284-7.

5. Zoghbi WA, Adams D, Bonow RO, Enriquez-Sarano M, Foster E, Grayburn PA, et al. Recommendations for noninvasive evaluation of native valvular
} 
regurgitation: A report from the American Society of Echocardiography developed in Collaboration with the Society for Cardiovascular Magnetic Resonance. J Am Soc Echocardiogr. 2017;30:303-71.

6. Zoghbi WA, Adams D, Bonow RO, Enriquez-Sarano M, Foster E, Grayburn PA, et al. Recommendations for noninvasive evaluation of native valvular regurgitation: a report from the American Society of Echocardiography developed in collaboration with the Society for Cardiovascular Magnetic Resonance. J Am Soc Echocardiogr. 2017;30:303-71

7. Said SM, Burkhart HM, Dearani JA. Surgical management of congenital (non-Ebstein) tricuspid valve regurgitation. Semin Thorac Cardiovasc Surg Pediatr Card Surg Annu. 2012;15:46-60.

8. Shinn SH, Schaff HV. Evidence-based surgical management of acquired tricuspid valve disease. Nat Rev Cardiol. 2013;10:190-203.

9. Hosmer DW, Lemeshow S, May S. Model development, applied survival analysis. In: Balding DJ, Cressie NAC, Fitzmaurice GM, eds. Regression Modeling of Time-to-Event Data. 2nd ed. Hoboken, NJ: John Wiley \& Sons, Inc; 2008:132-68.

10. Austin PC. Optimal caliper widths for propensity-score matching when estimating differences in means and differences in proportions in observational studies. Pharm Stat. 2011;10:150-61.

11. Al-Bawardy R, Krishnaswamy A, Bhargava M, Dunn J, Wazni O, Tuzcu EM, et al. Tricuspid regurgitation in patients with pacemakers and implantable cardiac defibrillators: a comprehensive review. Clin Cardiol. 2013;36:249-54.

12. Klutstein M, Balkin J, Butnaru A, Ilan M, Lahad A, Rosenmann D. Tricuspid incompetence following permanent pacemaker implantation. Pacing Clin Electrophysiol. 2009;32(suppl 1):S135-7.

13. Lin G, Nishimura RA, Connolly HM, Dearani JA, Sundt TM, Hayes DL. Severe symptomatic tricuspid valve regurgitation due to permanent pacemaker or implantable cardioverter-defibrillator leads. J Am Coll Cardiol. 2005;45: 1672-5.

14. Uehara K, Minakata K, Watanabe K, Sakaguchi H, Yamazaki K, Ikeda T, et al. Tricuspid valve repair for severe tricuspid regurgitation due to pacemaker leads. Asian Cardiovasc Thorac Ann. 2016;24:541-5.

15. Nucifora G, Badano LP, Allocca G, Gianfagna P, Proclemer A, Cinello M, et al. Severe tricuspid regurgitation due to entrapment of the anterior leaflet of the valve by a permanent pacemaker lead: role of real time three-dimensional echocardiography. Echocardiography. 2007;24:649-52.

16. Chen TE, Wang CC, Chern MS, Chu JJ. Entrapment of permanent pacemaker lead as the cause of tricuspid regurgitation. Circ J. 2007;71: 1169-71.

17. Glover BM, Watkins S, Mariani JA, Yap S, Asta J, Cusimano RJ, et al. Prevalence of tricuspid regurgitation and pericardial effusions following pacemaker and defibrillator lead extraction. Int J Cardiol. 2010;145:593-4.

18. Franceschi F, Thuny F, Giorgi R, Sanaa I, Peyrouse E, Assouan X, et al. Incidence, risk factors, and outcome of traumatic tricuspid regurgitation after percutaneous ventricular lead removal. J Am Coll Cardiol. 2009;53: 2168-74.

19. Nazmul MN, Cha YM, Lin G, Asirvatham SJ, Powell BD. Percutaneous pacemaker or implantable cardioverter-defibrillator lead removal in an attempt to improve symptomatic tricuspid regurgitation. Europace. 2013;15: 409-13.

20. Singh SK, Tang GH, Maganti MD, Armstrong S, Williams WG, David TE, et al. Midterm outcomes of tricuspid valve repair versus replacement for organic tricuspid disease. Ann Thorac Surg. 2006;82:1735-41.

21. Filsoufi F, Anyanwu A, Salzberg SP, Frankel T, Cohn LH, Adams DH. Long-term outcomes of tricuspid valve replacement in the current era. Ann Thorac Surg. 2005;80:845-50.

22. Ratnatunga CP, Edwards M, Dore CJ, Taylor KM. Tricuspid valve replacement: UK heart valve registry mid-term results comparing mechanical and biological prostheses. Ann Thorac Surg. 1998;66:1940-7.

23. Said SM, Dearani JA, Burkhart HM, Connolly HM, Eidem B, Stensrud PE, et al. Management of tricuspid regurgitation in congenital heart disease: is survival better with valve repair? J Thorac Cardiovasc Surg. 2014;147:412-7.

24. Moraca RJ, Moon MR, Lawton JS, Guthrie TJ, Aubuchon KA, Moazami N, et al. Outcomes of tricuspid valve repair and replacement: a propensity analysis. Ann Thorac Surg. 2009;87:83-8.

Key Words: permanent pacemaker, tricuspid valve, tricuspid valve regurgitation

\section{Discussion}

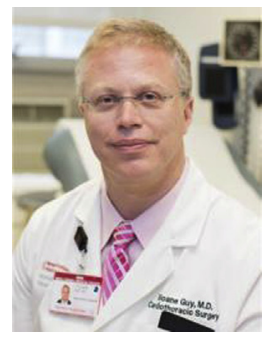

Dr T. S. Guy (New York, NY). What excited me about this article was that they say the TV is the forgotten valve. This is actually a common problem. Your stated incidence of TV regurgitation with pacemakers was high, and when you consider the number of pacemakers that are put in, this is a common clinical problem that at first may seem mundane but in fact is a large clinical issue. So I congratulate you on focusing on it.

There were a couple of questions that came out of my reading of your article. One was that you found that involvement of the PL predicted a higher incidence of TV replacement, and that puzzles me, because it's the smallest of the leaflets and in fact you don't even need it. You can turn the valve into a bicuspid valve by doing an annular plication in that area. It would seem to me that would be one of the easier valves to repair. Can you explain that?

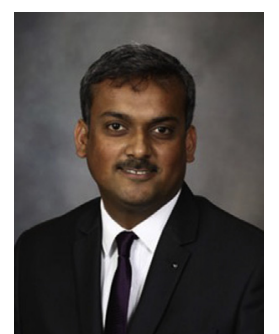

Dr N. Saran (Rochester, MN). I completely agree with that assessment, and that startled us as well. But when we looked deeper, we found that of the 42 patients who had PL involvement alone, only 9 of them had restricted mobility of the leaflet as the culprit mechanism; the rest all had scarring, torn leaflets, or perforation. It seemed from the operative findings that when the PL alone was involved, the leaflet was distorted to an extent that made repair not possible and could have acted as a deterrent to attempt repair.

It could also be possible that the distortion of the anatomy prevented the anterior and the SL to come together, which also could be related to a more extensive involvement of the subvalvar apparatus that might not have been noticed during surgery. Some of the surgeons tried Kay's annuloplasty, but it didn't seem to work possibly because of the reasons already mentioned. This finding was surprising, and I agree that this should make us think about alternate reasons or some findings that might have been missed. I would also like to emphasize that we must attempt repair in such patients rather than giving it up.

Dr Guy. That's very interesting. It also suggests that there may be in fact a problem with the other 2 leaflets.

Dr Saran. I agree and perhaps involvement of subvalvar apparatus as well.

Dr Guy. Because you would assume that if the anterior leaflet and SL were relatively normal and pliable that no matter what happened to the PL you should be able to repair that. There must be some involvement of the other 2 leaflets at least adjacent to the PL. 
Dr Saran. I agree.

Dr Guy. My second question is this. You found that TV replacement was associated with decreased survival. Why is that? Was it due to preoperative factors that were associated with TV replacement, is it the replacement itself, the age-old question, because in your conclusions you suggest that perhaps a more aggressive approach to repair is warranted, which somewhat implies that by repairing that valve you could expect a better outcome. So what is the causality that is leading to that result?

Dr Saran. When we looked into the multivariate analysis and controlled for factors such as diabetes, renal failure, dialysis, coronary artery disease, other preoperative factors, and concomitant cardiac surgery, we found that TV replacement was itself an independent risk factor for mortality.

Now, one of the reasons for TV replacement, of course, was that the leaflet was beyond repair and these patients may in general represent a poorer population subgroup with increased morbidity and features of right heart failure, but despite controlling for an exhaustive list of preoperative factors, we found that TV replacement was still an independent marker of poor prognosis.

I had also looked into our larger population subset in Mayo where we compared TV repair with replacement, and we still found that replacement had a worse prognosis. Why? The reasons could be various, the subset of patients itself, the right ventricle pathology involved, the right ventricular failure subsequent to replacement. We also lacked information regarding TV annulus size and right ventricle size, factors that could affect long-term survival. This topic requires a deeper analysis and remains open to discussion.

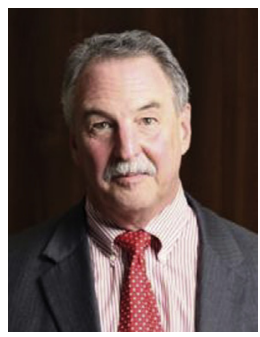

Dr J. Edgerton (Dallas, Tex). I want to congratulate you on an eloquent study. There is so much information in what you presented, and it is a sobering analysis of an increasingly prevalent iatrogenic problem that we face.

Because I regard the transvascular lead as the bane of the TV, I have tended in these cases to cut that lead off and get rid of it, and replace it with an epicardial left ventricular lead. This approach is also in keeping with my bias that left ventricular pacing is better anyway. The tradeoff, of course, is the potential for exit block. If when the left ventricular lead is placed, there is any trauma at all, then subsequent scarring occurs and leads to exit block. You referenced this technique to which I refer toward the end. Would you care to comment further?

Dr Saran. In approximately $2.5 \%$ of our patients, we excised the leads and substituted them with epicardial leads, but I agree that we need to look into this matter in more detail and try avoiding endocardial pacing leads, because, in a sense, these patients tend to do really poorly. So I agree completely that we need to have an alternative here in mind.

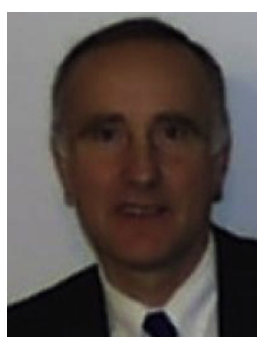

Dr G. Pettersson (Cleveland, Ohio). I agree with most of what you said. I want to ask you if we can firmly say to the electrophysiologists that there is no place for closed removal of leads in patients with severe TR?

Dr Bakaeen. So no lead extractions, only surgical interventions?

Dr Pettersson. No lead closed extractions in patients with important TR. You are only going to make TR worse.

Dr Saran. I completely agree with you. Now, there have been studies that say that lead extraction itself leads to TR and in such patients even more so.

Dr Pettersson. We can say that if there already is important TR, don't do closed lead extraction. Always be aware that whenever you do closed lead extract, you run the risk of damaging the TV and causing TR.

Dr Saran. I agree.

Dr Bakaeen. Show of hands, who agrees with this comment?

(Show of hands).

Dr Bakaeen. Some agree, I guess.

Dr Guy. I have had that situation with patients referred to you after they have attempted lead removal in hopes of fixing the TR, and it never works.

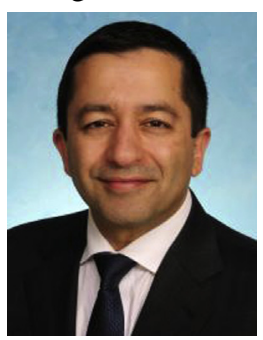

Dr V. Badhwar (Morgantown, WVa). I would caution the interpretation of tricuspid replacement being the source or item of causality for mortality. This is particularly given the lack of right ventricular size and other factors that you did not discuss. A lot of these patients have burnt out TR, and replacement is usually the procedure of choice, so I would be cautious in the interpretation.

Dr Saran. I agree. 
Original Sample $(n=598)$

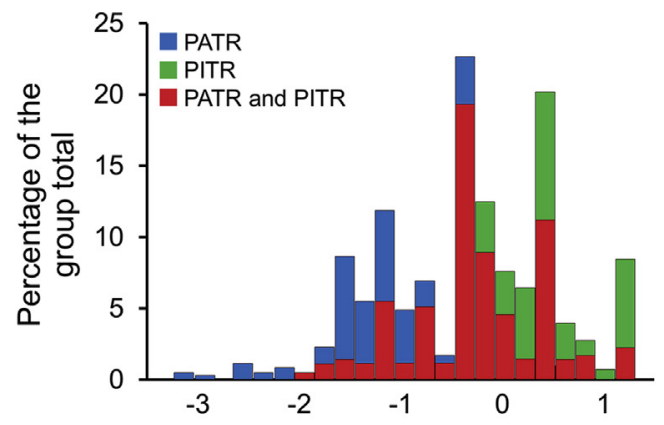

Propensity Score-Matched Subset $(n=406)$

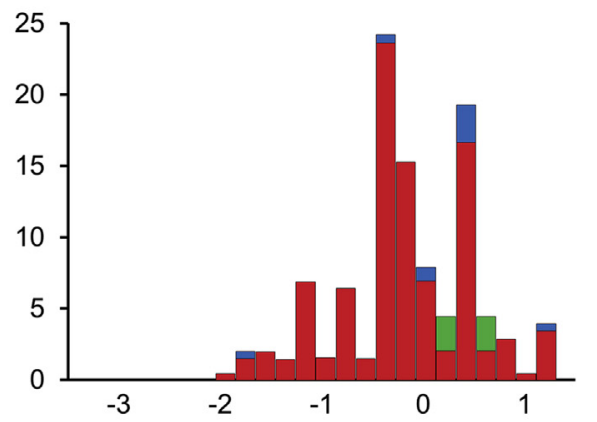

Distribution of propensity score

FIGURE E1. Propensity score distribution of the comprehensive and case-matched series. PATR, Pacemaker-associated tricuspid regurgitation; PITR, pacemaker-induced tricuspid regurgitation.

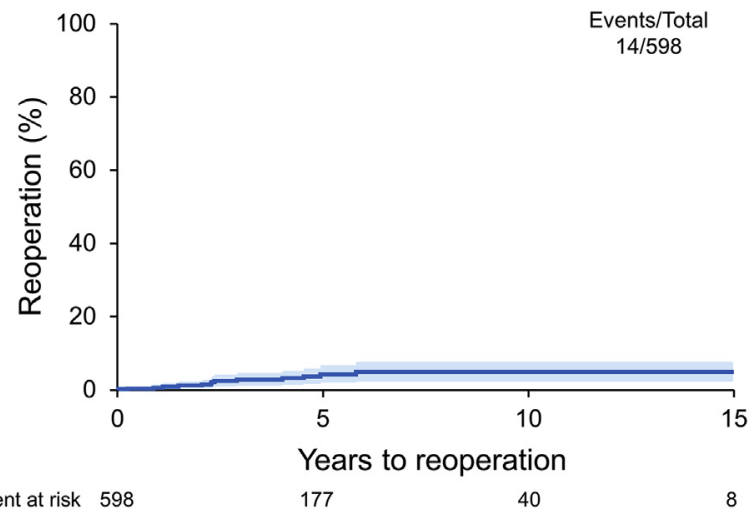

Patient at risk 598

177

40

FIGURE E2. Kaplan-Meier curve of incidence of reoperation in patients with PPM undergoing TV surgery. 


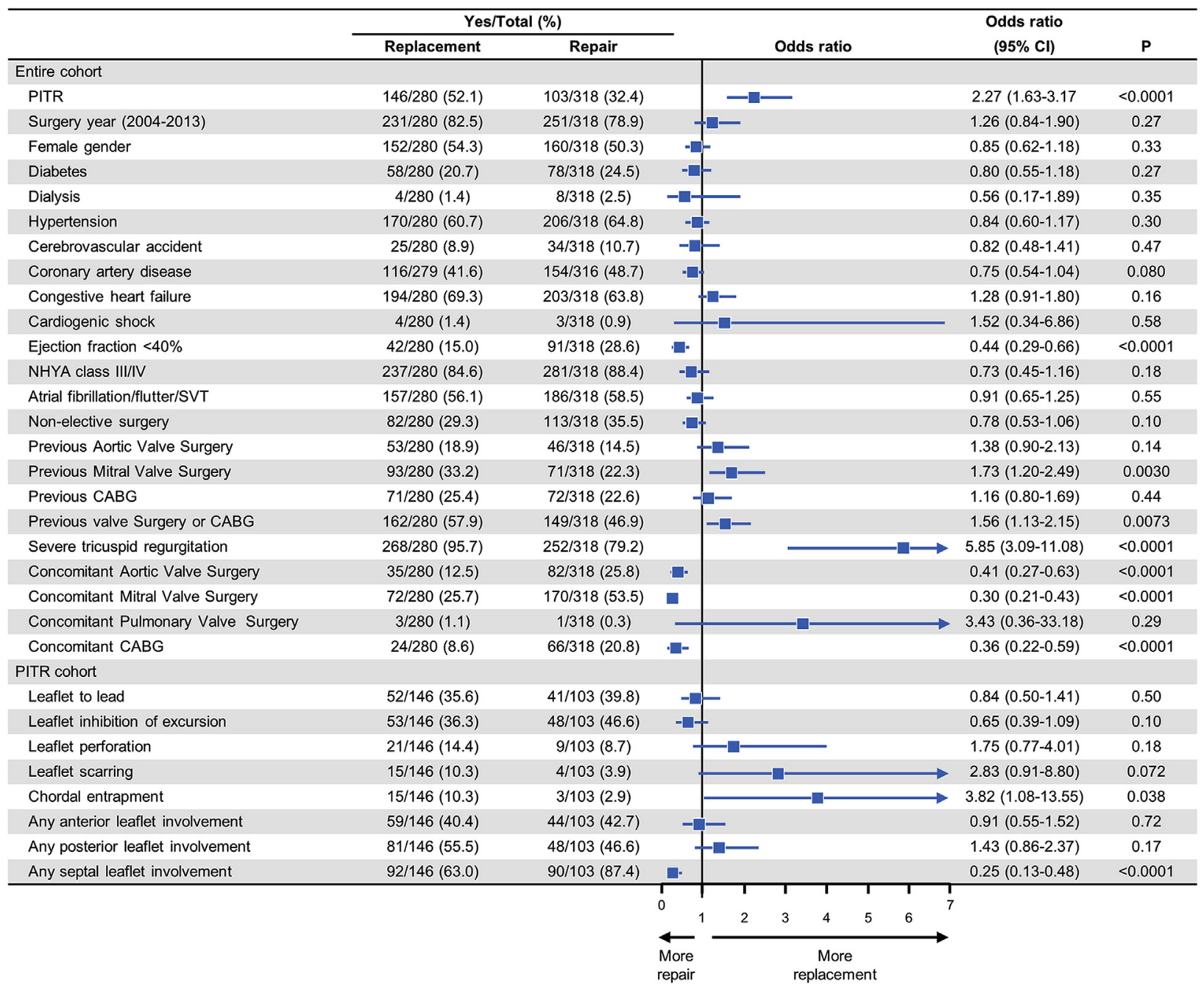

FIGURE E3. Univariate logistic regression predicting TV replacement (Forest plot). CI, Confidence interval; PITR, pacemaker-induced tricuspid regurgitation; NYHA, New York Heart Association; SVT, supraventricular tachycardia; $C A B G$, Coronary artery bypass grafting. 
TABLE E1. Risk factors for tricuspid valve replacement in patients with permanent pacemaker undergoing tricuspid valve surgery (multivariable analysis)

\begin{tabular}{|c|c|c|c|c|}
\hline Risk factor & Reference & OR & 95\% OR Confidence limits & $P$ value \\
\hline PITR & PATR & 2.141 & $1.440-3.184$ & .0002 \\
\hline Ejection fraction $<40 \%$ & No & 0.271 & $0.158-0.465$ & $<.0001$ \\
\hline Nonelective surgery & No & 0.683 & $0.430-1.085$ & .1067 \\
\hline NHYA III/IV & No & 0.661 & $0.365-1.198$ & .1725 \\
\hline Congestive heart failure & No & 1.897 & $1.208-2.977$ & .0054 \\
\hline Severe TR & Moderate & 5.387 & $2.482-11.691$ & $<.0001$ \\
\hline Previous MV surgery & No & 1.576 & $0.999-2.488$ & .0505 \\
\hline Previous TV surgery (repair) & No & 13.156 & $3.191-54.240$ & .0004 \\
\hline Concomitant mitral surgery & No & 0.262 & $0.172-0.397$ & $<.0001$ \\
\hline Concomitant aortic surgery & No & 0.329 & $0.197-0.548$ & $<.0001$ \\
\hline Concomitant CABG surgery & No & 0.401 & $0.223-0.721$ & .0023 \\
\hline Concomitant arrhythmia surgery & No & 0.493 & $0.250-0.971$ & .0409 \\
\hline Male & Female & 1.346 & $0.896-2.022$ & .1524 \\
\hline Cardiogenic shock & No & 3.310 & $0.612-17.903$ & .1646 \\
\hline Age & $10 \mathrm{y}$ & 1.186 & $1.002-1.402$ & .0469 \\
\hline
\end{tabular}

